\title{
Treatment and comorbidities of multiple sclerosis in an employed population in Japan: analysis of health claims data
}

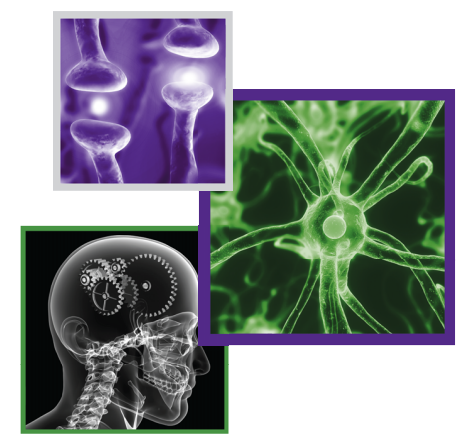

\author{
Mieko Ogino', Aki Shiozawa*,2, Hiroyuki Ota ${ }^{3}$, Shuichi Okamoto3, Shinzo Hiroi ${ }^{3}$ \& Izumi \\ Kawachi ${ }^{4}$ \\ ${ }^{1}$ International University of Health \& Welfare, School of Medicine, Center for Medical Education, 4-2 Kozunomori, Narita, Chiba, \\ 286-8686, Japan \\ ${ }^{2}$ Takeda Pharmaceuticals International Inc., 1 Takeda Parkway, Deerfield, IL 60015, USA \\ ${ }^{3}$ Japan Medical Affairs - Japan, Takeda Pharmaceutical Company Ltd, 12-10, Nihonbashi 2-chome, Chuo-ku, Tokyo, Japan \\ ${ }^{4}$ Department of Neurology, Brain Research Institute, Niigata University, 1-757 Asahimachidori, Chuo-ku, Niigata 951-8585, Japan \\ *Author for correspondence: aki.shiozawa@takeda.com
}

\section{Summary points}

- Data on the treatment, associated comorbidities and prevalence of multiple sclerosis (MS) are lacking in Japan.

- Data from the Japanese Medical Data Center health insurance claims database were analyzed from January 2005 to November 2014.

- Patients with a diagnosis claim for MS were identified using International Statistical Classification of Diseases and Related Health Problems, 10th Revision code G35 (MS).

- A total of 494 patients with MS were identified and associated comorbidities and prescribed treatments were investigated.

- IFN- $\beta$ and prednisolone were the most frequently prescribed treatments to prevent relapse.

- The prevalence of MS in an insured, employed Japanese population and their dependents increased between 2011 and 2013.

- The most common comorbidities identified were astigmatism, gastric ulcer, gastroesophageal reflux disease and low back pain.

- Some comorbidities were considered to be related to codes of reimbursement in the claims database rather than MS itself.

Aim: Use real-world data to investigate the treatment, comorbidities and prevalence of multiple sclerosis (MS) in an employed Japanese population. Methods: Data from the Japanese Medical Data Center health insurance claims database were analyzed (January 2005-November 2014). Patients with claims associated with an MS diagnosis were identified $(n=494)$ and comorbidities and prescribed treatments were investigated. MS prevalence within the database was calculated for each calendar year (2011-2013) Results: IFN- $\beta$ and prednisolone were the most commonly prescribed treatments. Common comorbidities included astigmatism and gastric ulcer. Within the database, MS prevalence increased from 0.015 to $0.016 \%$. Conclusion: MS prevalence increased from 2011 to 2013. Some comorbidities were considered unrelated to MS owing to how physicians use disease codes for reimbursement.

First draft submitted: 20 October 2017; Accepted for publication: 22 December 2017; Published online: 25 April 2018

Keywords: claims database $\bullet$ comorbidity $\bullet$ Japan $\bullet$ multiple sclerosis $\bullet$ prevalence $\bullet$ treatment

Multiple sclerosis (MS) is a chronic inflammatory disorder of the central nervous system and is estimated to affect 2.3 million individuals globally, although prevalences vary extensively across different countries and geographical regions [1]. The condition is noted to be the most frequent cause of nontraumatic neurological disability in young adults, requiring lifelong management with disease-modifying therapies [2]. Treatment to reduce the frequency of relapses, in particular, has been shown to slow disease progression [3-5]. Comorbidities including depression, anxiety 
and hypertension are common, and may also require specific management [6]. If not managed appropriately, MS may have implications for work capacity and health-related quality of life [7].

Data on the prevalence and treatment of MS, and associated comorbidities are particularly limited in Japan. The most recent of four nationwide surveys reported a fourfold increase in the estimated number of patients with MS between 1972 (2280 patients) and 2004 (9900 patients) [8-10], suggesting that the prevalence is increasing over time. A recent systematic review reported that comorbidity in MS is common, but highlighted substantial gaps in the epidemiological knowledge of comorbidity in patients with MS in Asia [11]. Data from the Japan 2014 National Health and Wellness Survey indicated that people with MS experience significantly greater overall work impairment than those without MS [12].

To gain a comprehensive understanding of trends associated with the treatment of a condition such as MS, it is essential to have access to real-world data that are representative of a given population. Health insurance claims databases document all medical treatments received by insured individuals across multiple treatment facilities. Access to these data allows the analysis of patient characteristics, treatments and costs for an extensive spectrum of medical conditions. The broad geographical coverage of this type of database also allows for the estimation of disease prevalence within the employed, medically insured population and their dependents.

The objective of this study was to use real-world data from a Japanese health insurance claims database to investigate the treatment, comorbidities and prevalence of MS in an employed population in Japan. Patients with MS who received public assistance with medical costs owing to disability, advanced age and poverty were not included in this analysis, nor were patients with progressive MS who did not receive treatment to prevent relapse.

\section{Materials \& methods}

\section{Data source}

Data for this study were obtained from the Japan Medical Data Center (JMDC) claims database, which contains retrospective claims data sourced from the Japanese union-managed health insurance system (the Japan Health Insurance Association). The database has broad geographical coverage with data available from multiple insurance providers across Japan, enabling the calculation of disease prevalence in the employed, medically insured population and their dependents. The database contains data from approximately 3 million individuals who are mainly employees of general corporations (i.e., individuals $<65$ years old) and their dependents. Patient eligibility information (e.g., start and stop dates of health plan enrollment), inpatient and outpatient medical claims (e.g., diagnosis codes, procedure codes, provider specialty, costs), pharmacy claims (e.g., drug name, cost) and annual health check-up data are captured in the JMDC. The database assigns each insured individual a unique identification number. All data were certified as anonymous and compliant with guidelines for the proper handling of personal information issued by the Japanese Ministry of Health, Labor and Welfare [13]. This study is reported in accordance with the Strengthening the Reporting of Observational Studies in Epidemiology guidelines.

\section{Patient selection}

Diagnosis claims were examined between 1 January 2005 and 30 November 2014. Approximately 3 million individuals were included in the database during this period. Data were extracted for all patients with a diagnosis claim for MS, which was identified using the International Statistical Classification of Diseases and Related Health Problems, 10th Revision (ICD-10) code G35 (MS). The study population was then identified using the algorithm in Table 1. Patients with a claim associated with an MS diagnosis were identified in the database. Those with a diagnosis claim for MS who also had a claim associated with a neuromyelitis optica (NMO) diagnosis (Japanese Standard Disease-Code 3410003) were excluded. Patients were included in the study if they met any of the following criteria: at least one hospitalization claim associated with a diagnosis of MS; at least one outpatient claim plus a claim for relapse treatment associated with a diagnosis of MS; at least one outpatient claim associated with a definitive diagnosis of MS and a diagnostic start date claim prior to the observation period; and three or more outpatient claims associated with a diagnosis of MS. There was no age limit for inclusion in the study.

\section{Study measures \\ Treatment to prevent relapse}

The proportions of patients in the study population prescribed at least one of seven treatments currently used in Japan to prevent relapse [14] were investigated from December 2009 to August 2014. Specific medications were identified in the database using the Anatomical Therapeutic Chemical classification system [15]. Treatment to 
Table 1. Algorithm for the identification of patients with multiple sclerosis.

\begin{tabular}{|c|c|c|}
\hline Inclusion/exclusion criteria & Number of patients, $\mathrm{n}(\%)$ & Female, \% \\
\hline Claim associated with MS diagnosis & $577(100)$ & 62 \\
\hline With claim associated with NMO diagnosis & $47(8)$ & 79 \\
\hline 1. At least one hospitalization claim associated with a diagnosis of MS & $202(35)$ & 54 \\
\hline $\begin{array}{l}\text { 2. At least one outpatient claim plus a claim for relapse treatment associated with a diagnosis of } \\
\text { MS (other than 1) }\end{array}$ & $129(22)$ & 67 \\
\hline $\begin{array}{l}\text { 3. At least one outpatient claim associated with a definitive diagnosis of MS and a diagnostic } \\
\text { start date claim prior to the observation period; (other than } 1 \text { and } 2 \text { ) }\end{array}$ & $163(28)$ & 69 \\
\hline 4. Three or more outpatient claims associated with a diagnosis of MS (other than 1, 2 and 3 ) & $0(0)$ & $\mathrm{n} / \mathrm{a}$ \\
\hline Total patients included $(1+2+3+4)$ & $494(86)$ & 62 \\
\hline $\begin{array}{l}\text { No claim associated with NMO diagnosis plus claim associated with MS diagnosis at least once } \\
\text { other than } 1+2+3+4\end{array}$ & $36(6)$ & 42 \\
\hline
\end{tabular}

prevent relapse was defined as at least one prescription of one of the following therapies after a diagnosis claim for MS: cyclophosphamide, azathioprine, tacrolimus, fingolimod, IFN- $\beta-1 \mathrm{a}$, IFN- $\beta-1 \mathrm{~b}$ or prednisolone. Prednisolone may be prescribed to patients in Japan to prevent relapse [14], and was therefore included in this analysis. In order to determine whether prescriptions from prednisolone were related to relapse or part of ongoing disease management, any prednisolone prescription made within 3 months of relapse was excluded. The date of relapse was defined as the date of first prescription of a drug to treat relapse symptoms occurring at least 30 days after a diagnosis claim for MS.

\section{Comorbidity analyses}

Comorbidities were identified using ICD-10 diagnosis codes. Comorbidity prevalences were calculated between January and November 2014 for patients with MS with available comorbidity data, and patients without a diagnosis claim for MS (i.e., all other patients who were insured) with available comorbidity data.

\section{MS prevalence within the employed, medically insured population \& their dependents}

The prevalence of MS within the JMDC claims database was calculated for each calendar year from 2011 to 2013; calculations accounted for the age distributions of patients with MS relative to that of insured individuals in the database who did not have MS. For each calendar year, patients with MS and insured individuals were stratified by age (0-9, 10-19, 20-29, 30-39, 40-49, 50-59, 60-69 and 70-79 years). Prevalences were then calculated using the following methodology: (1) the proportion of patients with MS relative to the total number of insured individuals was calculated for each age group; (2) the resulting values were multiplied by the total number of insured individuals in each age group; (3) the sum of the values for each age group from (2) was then divided by the sum of the total number of insured individuals in each age bracket to give the prevalence of MS for that calendar year. Prevalences were calculated separately for males and females.

\section{Statistical analyses}

Continuous variables were summarized by providing the mean, standard deviation (SD) and median. For categorical measures, data were presented as counts and proportions. Differences in the distribution of these variables were tested for statistical significance using Student's $t$-test. Statistical analyses were performed using SAS version 9.2 (SAS Institute, NC, USA).

\section{Results}

\section{Study participants \& baseline demographics}

A total of 577 patients had a diagnosis claim for MS between 1 January 2005 and 30 November 2014. Of these, 47 also had a diagnosis claim for NMO and were excluded from the analysis. A further 36 patients were excluded because they did not meet the criteria described in Table 1 . Of the 494 eligible patients (mean $\pm S D$ age $40.5 \pm 12.2$ years), 306 (62\%) were women. A total of 2,974,424 individuals (mean $\pm S D$ age $33.1 \pm 18.2$ years) with claims not associated with an MS diagnosis were insured between January 2005 and November 2014. 


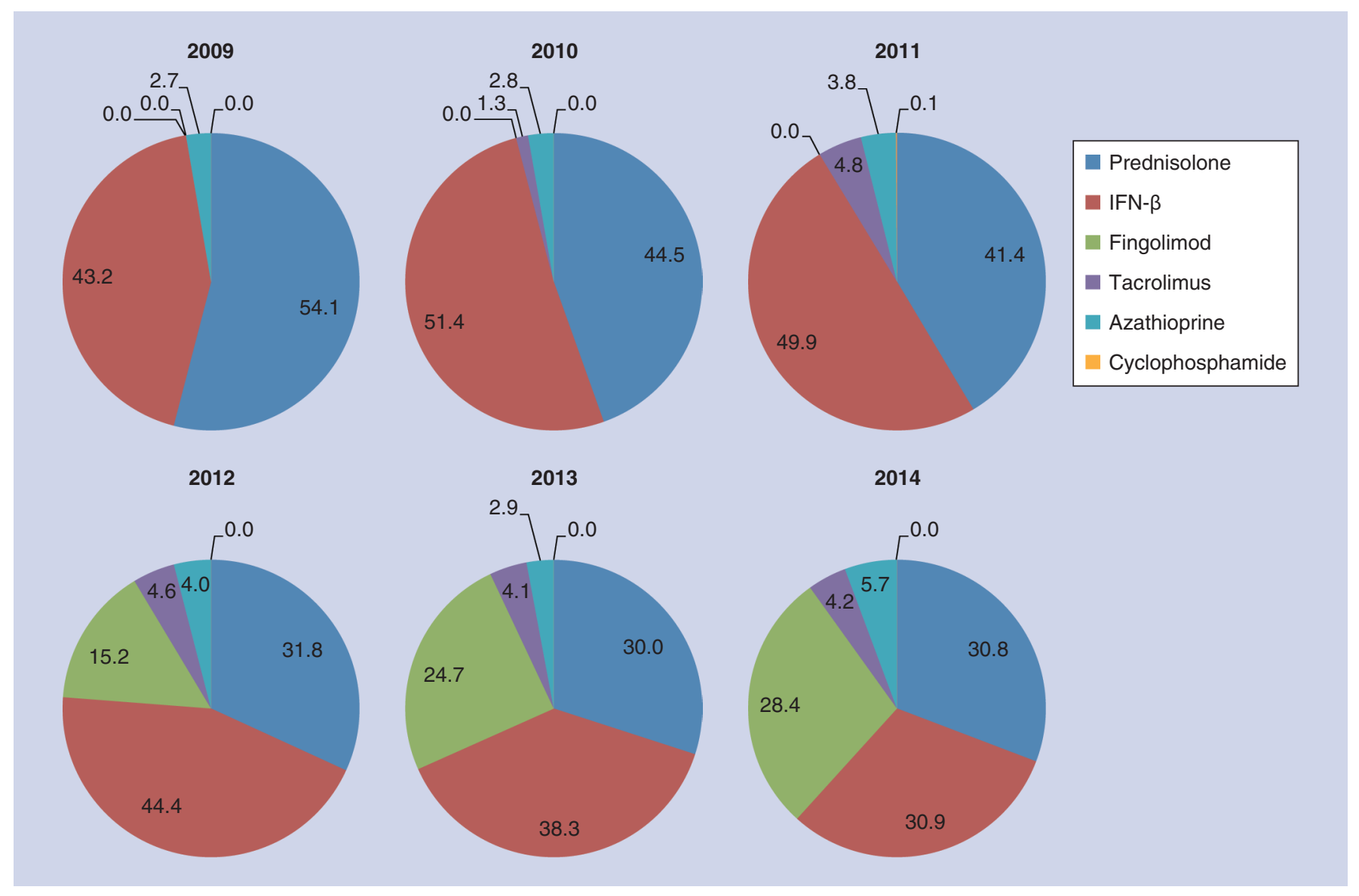

Figure 1. Percentage of patients with multiple sclerosis prescribed treatment to prevent relapse.

\section{Treatment to prevent relapse}

The proportions of patients who received at least one prescription for cyclophosphamide, azathioprine, tacrolimus, fingolimod, IFN- $\beta-1 \mathrm{a} / \mathrm{IFN}-\beta-1 \mathrm{~b}$ (IFN- $\beta$ ) or prednisolone from December 2009 to August 2014 are shown in Figure 1. Of the seven treatments investigated, IFN- $\beta$ and prednisolone were the most commonly prescribed, and the proportion of patients receiving these treatments remained consistent between 2009 and 2011 (Figure 1). The prescription of fingolimod increased between 2011 and November 2014, whereas the prescription of IFN- $\beta$ and prednisolone decreased during this period (Figure 1). Less than $10 \%$ of the patients were prescribed tacrolimus, azathioprine or cyclophosphamide; these proportions remained consistently low from 2009 to 2014 (Figure 1).

\section{Comorbidities}

A total of 235 patients with MS had available comorbidity data between January and November 2014. The most prevalent comorbidities in patients with MS were astigmatism (46\%), gastroesophageal reflux disease (GERD) with esophagitis (36\%), gastric ulcer (36\%), polyneuropathy (30\%) and constipation (30\%) (Table 2). In patients without a diagnosis claim for MS, astigmatism was the most common comorbidity $(21 \%)$, followed by hyperlipidemia (9\%), chronic gastritis (9\%) and low back pain (9\%). Prevalences of all identified comorbidities were significantly higher in patients with MS than in patients without MS $(\mathrm{p}<0.05)$ (Table 2).

\section{MS prevalence}

The prevalence of MS in the insured population within the JMDC for each calendar year between 2011 and 2013 is shown in Table 3. The prevalence of MS within this population increased from $0.015 \%$ in 2011 to $0.016 \%$ in 2013; however, the mean age of patients ( 43 years) remained consistent. The female:male ratio increased from 1:1.92 in 2011 to $1: 2.06$ in 2013. 


\begin{tabular}{|c|c|c|c|c|}
\hline ICD-10 code & Comorbidity & $\begin{array}{l}\text { Patients with MS } \\
(n=235)^{\dagger}\end{array}$ & $\begin{array}{l}\text { Patients without MS } \\
(\mathrm{n}=2,974,424)^{\ddagger}\end{array}$ & p-value \\
\hline E785 & Hyperlipidemia, unspecified & $59(25 \%)$ & $179,300(9 \%)$ & 0.0023 \\
\hline F329 & Major depressive disorder, single episode, unspecified & $39(17 \%)$ & $70,917(4 \%)$ & 0.0155 \\
\hline G409 & Epilepsy, unspecified & $40(17 \%)$ & $19,024(1 \%)$ & 0.0030 \\
\hline M545 & Low back pain & $69(29 \%)$ & $169,118(9 \%)$ & 0.0001 \\
\hline R51 & Headache & $37(16 \%)$ & $101,786(5 \%)$ & 0.0337 \\
\hline D509 & Iron deficiency anemia, unspecified & $38(16 \%)$ & $84,086(6 \%)$ & 0.0376 \\
\hline E14 & Unspecified diabetes mellitus & $38(16 \%)$ & $102,524(5 \%)$ & 0.0304 \\
\hline G470 & Insomnia & $64(27 \%)$ & $111,214(6 \%)$ & 0.0001 \\
\hline G629 & Polyneuropathy, unspecified & $71(30 \%)$ & $60,862(3 \%)$ & $<0.0001$ \\
\hline H522 & Astigmatism & $108(46 \%)$ & $510,600(21 \%)$ & $<0.0001$ \\
\hline K210 & GERD with esophagitis & $84(36 \%)$ & $134,037(7 \%)$ & $<0.0001$ \\
\hline K259 & $\begin{array}{l}\text { Gastric ulcer, unspecified as acute or chronic, without hemorrhage } \\
\text { or perforation }\end{array}$ & $84(36 \%)$ & $106,619(6 \%)$ & $<0.0001$ \\
\hline K295 & Unspecified chronic gastritis without bleeding & $52(22 \%)$ & $170,002(9 \%)$ & 0.0129 \\
\hline K590 & Constipation, unspecified & $70(30 \%)$ & $153,153(7 \%)$ & $<0.0001$ \\
\hline M478 & Other spondylosis & $39(17 \%)$ & $62,805(3 \%)$ & 0.0136 \\
\hline M819 & Osteoporosis without pathological fracture: site unspecified & $49(21 \%)$ & $28,997(2 \%)$ & 0.0005 \\
\hline
\end{tabular}

\begin{tabular}{|c|c|c|c|}
\hline \multirow[t]{2}{*}{ Variable } & \multicolumn{3}{|c|}{ Prevalence (\%) } \\
\hline & 2011 & 2012 & 2013 \\
\hline Male & 0.010 & 0.011 & 0.011 \\
\hline Female & 0.020 & 0.021 & 0.022 \\
\hline Total & 0.015 & 0.015 & 0.016 \\
\hline Female:male ratio & 1.92 & 1.83 & 2.06 \\
\hline Mean \pm SD age, years & $42.8 \pm 12.3$ & $42.5 \pm 11.4$ & $43.0 \pm 11.3$ \\
\hline
\end{tabular}

\section{Discussion}

This study used real-world data from a Japanese health insurance claims database to investigate the treatment, comorbidities and prevalence of MS in an employed population in Japan. Of the seven treatments investigated, IFN- $\beta$ and prednisolone were the most commonly used to prevent relapse, together accounting for over $90 \%$ of prescriptions before 2011. Following the regulatory approval of the oral treatment fingolimod in Japan in 2011 [19], the proportion of patients prescribed IFN- $\beta$ or prednisolone fell in parallel with an increase in the proportion prescribed fingolimod. Despite these changes, IFN- $\beta$ and prednisolone continued to account for approximately $60-70 \%$ of all prescriptions from 2012 to 2014.

In a recent systematic literature review, the most common comorbidities reported in prevalent MS cohorts were depression (23.7\%), anxiety (21.9\%), hypertension (18.6\%), hyperlipidemia (10.9\%) and chronic lung disease (10.0\%) [6]. In our employed study population, a lower prevalence of depression (17\%) and a higher prevalence of hyperlipidemia (25\%) were observed, and anxiety, hypertension and chronic lung disease were not recorded as comorbidities during the 11 month period studied. This may be owing to the fact that anxiety is not frequently related to reimbursement, and that hypertension and chronic lung disease are more frequently observed in older individuals who may be under-represented in the JMDC database [20,21].

The most prevalent comorbidities in our study population were astigmatism, gastric ulcer, GERD and low back pain. Owing to the claims-based nature of these data, it is likely that some comorbidities identified may not be 
directly related to MS. For example, the code for astigmatism (ICD-10 code H522) may be used by ophthalmologists following an eye examination during the diagnosis of MS. Similarly, the code for 'other spondylosis' (a degenerative spinal disorder; ICD-10 code M478) may be used for reimbursement following magnetic resonance imaging during the diagnosis of MS.

Our analysis identified an apparent increase in the prevalence of MS within the employed, medically insured population and their dependents from 2011 to 2013, which is consistent with previous small-scale epidemiological studies in Japan [16-18]. However, our estimates were based on claims associated with an MS diagnosis, and may not reflect trends in nationwide MS prevalence in Japan. Additional epidemiological studies with a longer observation period will help to determine if the prevalence of MS in the employed population in Japan is truly increasing.

The use of health claims data provided valuable information on annual trends in medical care associated with MS; however, there are limitations associated with analyses of this type. For example, the study population may not be representative of the Japanese population in terms of geographical distribution and age distribution. This study also excluded unemployed individuals who represented 29-41\% of the Japanese population from 2005 to 2014 [24]. Patients with MS were identified using ICD-10 diagnosis codes instead of conventional diagnostic procedures; these codes are subject to coding errors or incorrect diagnostic information and cannot be verified, which may have resulted in the inclusion of individuals without MS in the study population. Some patients with NMO may also have remained in the study population owing to the lack of available NMO-specific diagnostic tests during the study period [27]. In addition, ICD-10 codes assigned to patients before diagnosis of MS may remain in the database, making it challenging to identify comorbidities that were concomitant with MS. These challenges are expected to be overcome in future studies using more comprehensive databases.

\section{Conclusion}

This real-world claims database analysis furthers our knowledge of prescribed medications, potential comorbidities and the prevalence of MS in an insured, employed population and their dependents in Japan. Further research in a study population inclusive of unemployed individuals and those over the age of 65 years, is required to improve the understanding of the epidemiology of MS in Japan, and the impact of comorbidities associated with MS on overall patient burden.

\section{Acknowledgements}

The authors would like to thank K Iwasaki (Milliman, Japan) for providing assistance with statistical analyses.

\section{Financial \& competing interests disclosure}

A Shiozawa is an employee of Takeda Pharmaceuticals International Inc. H Ohta, S Okamoto and S Hiroi are the employees of Takeda Pharmaceutical Company Ltd. M Ogino and I Kawachi have received funding for travel from Takeda Pharmaceutical Company Ltd. The authors have no other relevant affiliations or financial involvement with any organization or entity with a financial interest in or financial conflict with the subject matter or materials discussed in the manuscript apart from those disclosed.

Medical writing support was provided by L Knapp PhD of PharmaGenesis London, London, UK.

Ethical conduct of research

The authors state that this study was compliant with guidelines for the proper handling of personal information issued by the Japanese Ministry of Health, Labour and Welfare.

\section{Open access}

This work is licensed under the Attribution-NonCommercial-NoDerivatives 4.0 Unported License. To view a copy of this license, visit http://creativecommons.org/licenses/by-nc-nd/4.0/

\section{References}

Papers of special note have been highlighted as: • of interest; $\bullet \bullet$ of considerable interest

1. Browne P, Chandraratna D, Angood C et al. Atlas of multiple sclerosis 2013: a growing global problem with widespread inequity. Neurology 83(11), 1022-1024 (2014).

2. Eskandarieh S, Heydarpour P, Minagar A, Pourmand S, Sahraian MA. Multiple sclerosis epidemiology in east Asia, south-east Asia and south Asia: a systematic review. Neuroepidemiology 46(3), 209-221 (2016).

3. Calabresi PA, Radue EW, Goodin D et al. Safety and efficacy of fingolimod in patients with relapsing-remitting multiple sclerosis (FREEDOMS II): a double-blind, randomised, placebo-controlled, Phase 3 trial. Lancet Neurol. 13(6), 545-556 (2014). 
4. Kappos L, Radue EW, O'Connor P et al. A placebo-controlled trial of oral fingolimod in relapsing multiple sclerosis. N. Engl. J. Med. 362(5), 387-401 (2010).

5. The IFNB Multiple Sclerosis Study Group. Interferon beta-1b is effective in relapsing-remitting multiple sclerosis. I. Clinical results of a multicenter, randomized, double-blind, placebo-controlled trial. Neurology 43(4), 655-661 (1993).

6. Marrie RA, Cohen J, Stuve $\mathrm{O}$ et al. A systematic review of the incidence and prevalence of comorbidity in multiple sclerosis: overview. Mult. Scler. 21(3), 263-281 (2015).

-. An in-depth review of the comorbidities of multiple sclerosis (MS).

7. Flensner G, Landtblom AM, Soderhamn O, Ek AC. Work capacity and health-related quality of life among individuals with multiple sclerosis reduced by fatigue: a cross-sectional study. BMC Public Health 13, 224-234 (2013).

8. Kuroiwa Y, Igata A, Itahara K, Koshijima S, Tsubaki T. Nationwide survey of multiple sclerosis in Japan. Clinical analysis of 1084 cases. Neurology 25(9), 845-851 (1975).

9. Osoegawa M, Kira J, Fukazawa T et al. Temporal changes and geographical differences in multiple sclerosis phenotypes in Japanese: nationwide survey results over 30 years. Mult. Scler. 15(2), 159-173 (2009).

-• Data on the geographical differences in MS phenotype from of a nationwide survey of MS in Japan over a 30-year period.

10. Shibasaki H, Kubo N, Nishitani $\mathrm{H}$ et al. Nationwide survey of multiple sclerosis in Japan: reappraisal of clinical features. J. Trop. Geo. Neurol. 2, 73-82 (1992).

11. Marrie RA, Reider N, Cohen J et al. A systematic review of the incidence and prevalence of sleep disorders and seizure disorders in multiple sclerosis. Mult. Scler. 21(3), 342-349 (2015).

12. Yamabe K, Witt EA, DiBonaventura M. The burden of multiple sclerosis in Japan. Value Health 19(3), A66 (2016).

13. Japanese Ministry of Health Labour and Welfare. Guidelines for the proper handling of personal information (2004). www.mhlw.go.jp/topics/bukyoku/seisaku/kojin/dl/161227kenpo.pdf

14. Japanese Society of Neurology, Japanese Society for Neuroimmunology, Japanese Society of Neurological Therapeutics. Guidelines for treatment of multiple sclerosis (2010). www.neurology-jp.org/guidelinem/koukasyo.html

15. WHO Collaborating Centre for Drug Statistics Methdology. ATC/DDD Index (2016). www.whocc.no/atc_ddd_index/

16. Houzen H, Niino M, Hata D et al. Increasing prevalence and incidence of multiple sclerosis in northern Japan. Mult. Scler. 14(7), $887-892$ (2008).

17. Houzen $\mathrm{H}$, Niino $\mathrm{M}$, Hirotani $\mathrm{M}$ et al. Increased prevalence, incidence, and female predominance of multiple sclerosis in northern Japan. J. Neurol. Sci. 323(1-2), 117-122 (2012).

-. A study of the epidemiology of MS in Japan and comparison with previous epidemiological studies in Japan.

18. Houzen H, Niino M, Kikuchi S et al. The prevalence and clinical characteristics of MS in northern Japan. J. Neurol. Sci. 211(1-2), 49-53 (2003).

19. Matsui M, Shimizu Y, Doi H et al. Japanese guidelines for fingolimod in multiple sclerosis: putting into practice. Clin. Exp. Neuroimmunol. 5, 34-48 (2014).

20. Miura K, Nagai M, Ohkubo T. Epidemiology of hypertension in Japan: where are we now? Circ. J. 77(9), 2226-2231 (2013).

21. Rycroft CE, Heyes A, Lanza L, Becker K. Epidemiology of chronic obstructive pulmonary disease: a literature review. Int. J. Chron. Obstruct. Pulmon. Dis. 7, 457-494 (2012).

22. Gartzen K, Katzarava Z, Diener HC, Putzki N. Peripheral nervous system involvement in multiple sclerosis. Eur. J. Neurol. 18(5), 789-791 (2011).

23. Organisation for Economic Cooperation and Development. Population (indicator) (2017). https://data.oecd.org/pop/population.htm

24. Organisation for Economic Cooperation and Development. Long-term unemployment rate in Japan 2005-2014. https://data.oecd.org/unemp/long-term-unemployment-rate.htm\#indicator-chart

25. Yokote H, Mizusawa H. Multiple sclerosis and neuromyelitis optica spectrum disorders: some similarities in two distinct diseases. Neural Regen. Res. 11(3), 410-411 (2016).

26. Wingerchuk DM, Lennon VA, Pittock SJ, Lucchinetti CF, Weinshenker BG. Revised diagnostic criteria for neuromyelitis optica. Neurology 66(10), 1485-1489 (2006).

27. Japanese Ministry of Health Labour and Welfare Central Social Insurance Medical Council meeting minutes 23 October 2013 (2013). www.mhlw.go.jp/stf/shingi/0000029035.html

28. Papadopoulos MC, Verkman AS. Aquaporin 4 and neuromyelitis optica. Lancet Neurol. 11(6), 535-544 (2012). 